\title{
Algebra Structure of Operator-Valued Riesz Means
}

\author{
Pedro J. Miana \\ Departamento de Matemáticas and I.U.M.A., Universidad de Zaragoza, 50009 Zaragoza, Spain \\ Correspondence should be addressed to Pedro J. Miana; pjmiana@unizar.es
}

Received 30 November 2013; Revised 2 March 2014; Accepted 27 March 2014; Published 18 May 2014

Academic Editor: Hagen Neidhardt

Copyright (C) 2014 Pedro J. Miana. This is an open access article distributed under the Creative Commons Attribution License, which permits unrestricted use, distribution, and reproduction in any medium, provided the original work is properly cited.

We characterize operator-valued Riesz means via an algebraic law of composition and establish their functional calculus accordingly. With this aim, we give a new integral expression of the Leibniz derivation rule for smooth functions.

\section{Introduction}

Every self-adjoint operator on a Hilbert space admits a spectral decomposition (on Borel subsets of its spectrum) which allows to define $L^{\infty}$-functional calculus. Spectral operators of scalar type (introduced in [1]) on arbitrary Banach spaces also have this functional calculus. If spectral projections (associated to a given operator) are required to be uniformly bounded only to closed intervals, these operators are said to be well-bounded. Roughly speaking, in reflexive Banach spaces $X$, a well-bounded operator $(A, D(A))$ with $\sigma(A) \subset$ $[0, \infty)$ admits a projection-valued function $E:[0, \infty) \rightarrow$ $\mathscr{B}(X)$ such that $E(t) \sim \chi_{(0, t)}(A)[1-3]$.

Well-bounded operators on $[0, \infty)$ have a functional calculus for absolutely continuous functions [2], and Riesz means, $G_{t}^{n}:=(1 / n !)(t-A)_{+}^{n}($ for $t>0$ and $n \in \mathbb{N} \cup\{0\})$, are properly defined by this functional calculus; in some sense, $G_{t}^{n}$ is the $n$-times integral of the decomposition of the identity associated to $A$; see [4, page 332$]$.

The aim of this paper is to introduce the operator-valued Riesz means $\left(G_{t}^{n}\right)_{t>0} \subset \mathscr{B}(X)$ (for $n \in \mathbb{N}$ ) in an axiomatic way taking into account the algebraic law of the composition $G_{t}^{n} G_{s}^{n}$, the uniform boundedness, and the summability property; see Definition 5. The starting point is a multiplication identity for scalar Riesz means (Proposition 1) and led us to a new expression to the Leibniz formula, Proposition 3. To conclude, we show that certain holomorphic $C_{0}$-semigroups, $A C^{(n)}$-functional calculus, and operator-valued Riesz means are equivalent concepts, essentially up some regularity; see Section 4 .
There exist alternative approaches to operator-valued Riesz means $\left(G_{t}^{v}\right)_{t>0}$, (for $\left.v>0\right)$ mainly closer to approximation theory and Fourier multipliers; see, for example, [5, page 193], [6, Section 2], [7, Section 3], and the references therein. Note that our point of view may be applied in some of these settings.

Notation. In this paper, $\mathbb{C}^{+}:=\{z \in \mathbb{C} \mid \mathfrak{R} z>0\} ; \chi_{E}$ is the characteristic function in the set $E$; we write $(s-|a|)_{+}=(s-$ $|a|) \chi_{(0, a)} . X$ is a Banach space, and $\mathscr{B}(X)$ is the set of linear and bounded operators on $X ;(A, D(A))$ is a closed operator on $X$, and $\sigma(A)$ is the spectrum of $A$.

\section{Riesz Functions}

For $n \in \mathbb{N}$, we consider Riesz functions $\left(R_{t}^{n}\right)_{t \geq 0}$ given by

$$
R_{t}^{n}(u):=\frac{(t-|u|)_{+}^{n}}{n !}, \quad u \in \mathbb{R}
$$

for $t>0$ and $R_{0}^{n}=0$. Note that $R_{t}^{0}=\chi_{(-t, t)}$ for $t>0$ and

$$
\begin{array}{r}
R_{t}^{n}(\xi)=\frac{1}{(n-j) !} \int_{0}^{t}(t-u)^{n-j} R_{u}^{j-1}(\xi) d u \\
\xi \in \mathbb{R}
\end{array}
$$

for $1 \leq j \leq n$. 
Proposition 1. Let $n \in \mathbb{N}$ and $t \geq s \geq 0$. Then,

$$
\begin{aligned}
R_{t}^{n} R_{s}^{n}= & \sum_{j=1}^{n}(t-s)^{n-j} \frac{(n+j) !}{n !^{2}}\left(\begin{array}{c}
n \\
j
\end{array}\right) \\
& \times \int_{0}^{s} \frac{(s-u)^{j-1}}{(j-1) !} R_{u}^{n} d u+\frac{(t-s)^{n}}{n !} R_{s}^{n} .
\end{aligned}
$$

Proof. Take $\xi \in \mathbb{R}$ such that $|\xi| \leq s$; note that

$$
\begin{aligned}
\sum_{j=1}^{n}( & -s)^{n-j} \frac{(n+j) !}{n !^{2}}\left(\begin{array}{c}
n \\
j
\end{array}\right) \int_{0}^{s} \frac{(s-u)^{j-1}}{(j-1) !} R_{u}^{n}(\xi) d u \\
& +\frac{(t-s)^{n}}{n !} R_{s}^{n}(\xi) \\
= & \sum_{j=1}^{n}(t-s)^{n-j} \frac{(n+j) !}{n !^{2}}\left(\begin{array}{c}
n \\
j
\end{array}\right) \\
& \times \int_{|\xi|}^{s} \frac{(s-u)^{j-1}}{(j-1) !} \frac{(u-|\xi|)^{n}}{n !} d u \\
& +\frac{(t-s)^{n}}{n !} \frac{(s-|\xi|)^{n}}{n !} \\
= & \frac{(s-|\xi|)^{n}}{n !} \\
& \times\left(\frac{1}{n !} \sum_{j=1}^{n}\left(\begin{array}{l}
n \\
j
\end{array}\right)(t-s)^{n-j}(s-|\xi|)^{j}+\frac{(t-s)^{n}}{n !}\right)
\end{aligned}
$$

For $|\xi| \geq s$, the equality holds trivially.

We consider functions $\left(f_{0, s}\right)_{s \geq 0}$ defined by

$$
f_{0, s}(r):=\frac{\sin (s r)}{\pi r}, \quad r \in \mathbb{R}
$$

and now define functions $\left(f_{n, s}\right)_{s \geq 0}$ by integration:

$$
f_{n, s}(r):=\frac{1}{(n-1) !} \int_{0}^{s}(s-u)^{n-1} f_{0, u}(r) d u
$$

for $n \in \mathbb{N}$ and $s \geq 0$. It is straightforward to prove that

$$
\begin{aligned}
& f_{2 m, s}(r) \\
& =\frac{1}{\pi r^{2 m+1}}\left((-1)^{m} \sin (r s)\right. \\
& \left.\quad+\sum_{j=0}^{m-1}(-1)^{j} \frac{(r s)^{2(m-j)-1}}{(2(m-j)-1) !}\right), \\
& f_{2 m-1, s}(r) \frac{1}{\pi r^{2 m}}\left(\begin{array}{c}
(-1)^{m} \cos (r s) \\
\left.\quad+\sum_{j=0}^{m-1}(-1)^{j} \frac{(r s)^{2(m-1-j)}}{(2(m-1-j)) !}\right),
\end{array}\right.
\end{aligned}
$$

for $r \in \mathbb{R}, m \geq 1$, and $s \geq 0$. Note that these functions, $f_{2 m, s}$ and $f_{2 m-1, s}$, up some factors, are the remainders of the Taylor series of $\sin (\cdot s)$ and $\cos (\cdot s)$, respectively.

Note that $f_{n, s} \in L^{1}(\mathbb{R})$ for $n \in \mathbb{N}$ and $s \geq 0$. We remind the reader that the Fourier transform of a function $f$ in $L^{1}(\mathbb{R})$ is defined by

$$
\mathscr{F} f(t):=\int_{-\infty}^{\infty} e^{-i x t} f(x) d x, \quad t \in \mathbb{R} .
$$

It is well known that $\mathscr{F} f$ is continuous on $\mathbb{R}$ and $\mathscr{F} f(t) \rightarrow 0$ when $|t| \rightarrow \infty$ (the Riemann-Lebesgue lemma). In the case that $f \in L^{p}(\mathbb{R})$ for some $1<p \leq 2$, the Fourier transform of $f$ is defined in terms of a limit in the norm of $L^{p^{\prime}}(\mathbb{R})$ of truncated integrals:

$$
\begin{gathered}
\mathscr{F} f:=\lim _{R \rightarrow \infty} \mathscr{F}\left(f \chi_{(-R, R)}\right), \\
\mathscr{F}\left(f \chi_{(-R, R)}\right)(t)=\int_{-R}^{R} e^{-i x t} f(x) d x, \quad t \in \mathbb{R} ;
\end{gathered}
$$

that is, $\mathscr{F} f \in L^{p^{\prime}}(\mathbb{R})$ and $\lim _{R \rightarrow \infty}\left\|\mathscr{F} f-\mathscr{F}\left(f \chi_{(-R, R)}\right)\right\|_{p^{\prime}}=$ 0 , where $1 / p+1 / p^{\prime}=1$ and $\chi_{(-R, R)}$ is the characteristic function of the interval $(-R, R)$; see, for example, [8, Volume 2 , page 254]. Then, the existence of $\mathscr{F} f(t)$ is guaranteed only at almost every $t$, and $\mathscr{F} f$ may be noncontinuous and the Riemann-Lebesgue lemma could not hold (unlike the case when $\left.f \in L^{1}(\mathbb{R})\right)$.

Remark 2. For $n \in \mathbb{N}$ and $s \geq 0$, we have $\mathscr{F}\left(f_{n, s}\right)=R_{s}^{n}$ and

$$
\begin{aligned}
f_{n, t} * f_{n, s}= & \sum_{j=1}^{n}(t-s)^{n-j} \frac{(n+j) !}{n !^{2}}\left(\begin{array}{c}
n \\
j
\end{array}\right) \\
& \times \int_{0}^{s} \frac{(s-u)^{j-1}}{(j-1) !} f_{n, u} d u+\frac{(t-s)^{n}}{n !} f_{n, s},
\end{aligned}
$$


for $t, s \geq 0$. To show this, note that $\mathscr{F}\left(f_{0, s}\right)=\chi_{(-s, s)}$ a.e., and then we have that

$$
\begin{aligned}
\mathscr{F}\left(f_{n, s}\right)(\xi) & =\frac{1}{(n-1) !} \int_{0}^{s}(s-u)^{n-1} \chi_{(-u, u)}(\xi) d u \\
& =\frac{(s-|\xi|)_{+}^{n}}{n !}=R_{s}^{n}(\xi)
\end{aligned}
$$

for $\xi \in \mathbb{R}, n \in \mathbb{N}$, and $s \geq 0$. We apply the Fourier transform and Proposition 1 to get equality (10).

\section{Leibniz Formula via Integrals}

In this section, we give a new expression of the Leibniz formula $(f g)^{(n)}$ for functions $f, g \in \mathcal{S}$, where the set $\mathcal{S}$ is the Schwartz class of functions on $[0, \infty)$.

Proposition 3. For $n \geq 2$ and $f, g \in \mathcal{S}$, one has that

$$
\begin{aligned}
(f g)^{(n)}(t)= & f^{(n)}(t) g(t)+f(t) g^{(n)}(t) \\
& +\sum_{j=1}^{n-1}\left(\begin{array}{c}
n+j-1 \\
j
\end{array}\right) \\
& \times \int_{t}^{\infty}(-1)^{j} \frac{(u-t)^{j-1}}{(j-1) !} \\
& \quad \times\left(f^{(n)}(u) g^{(j)}(u)+f^{(j)}(u) g^{(n)}(u)\right) d u
\end{aligned}
$$

for $t \geq 0$.

Proof. We write by $H_{n}(f, g)(t)$ the right part of equality (12). We prove the formula by the induction method. For $n=2$, we have that

$$
\begin{aligned}
& f^{(2)}(t) g(t)+f(t) g^{(2)}(t) \\
& \quad-2 \int_{t}^{\infty}\left(f^{(2)}(u) g^{(1)}(u)+f^{(1)}(u) g^{(2)}(u)\right) d u \\
& =f^{(2)}(t) g(t)+f(t) g^{(2)}(t)+2 f^{(1)}(t) g^{(1)}(t) \\
& =(f g)^{(2)}(t)
\end{aligned}
$$

for $t \geq 0$. Now, we suppose that $(f g)^{(n)}=H_{n}(f, g)$, and we will show that $\left(H_{n}(f, g)\right)^{\prime}=H_{n+1}(f, g)$, where we conclude that $(f g)^{(n+1)}=H_{n+1}(f, g)$. By the induction method, we conclude equality (12).
For $t \geq 0$, we have that

$$
\begin{aligned}
& \left(H_{n}(f, g)\right)^{(1)}(t) \\
& =f^{(n+1)}(t) g(t)+f(t) g^{(n+1)}(t) \\
& \quad+(n+1)\left(f^{(n)} g^{(1)}+f^{(1)} g^{(n)}\right)(t) \\
& \quad+\sum_{j=2}^{n-1}\left(\begin{array}{c}
n+j-1 \\
j
\end{array}\right) \\
& \quad \times \int_{t}^{\infty}(-1)^{j+1} \frac{(u-t)^{j-2}}{(j-2) !} \\
& \quad \times\left(f^{(n)}(u) g^{(j)}(u)+f^{(j)}(u) g^{(n)}(u)\right) d u .
\end{aligned}
$$

Note that, for $1 \leq j \leq n$, we obtain that

$$
\begin{aligned}
f^{(n)}(u) g^{(j)}(u) & =-\int_{u}^{\infty}\left(f^{(n)} g^{(j)}\right)^{(1)}(s) d s \\
& =-\int_{u}^{\infty}\left(f^{(n+1)} g^{(j)}+f^{(n)} g^{(j+1)}\right)(s) d s
\end{aligned}
$$

for $u \geq 0$, and then

$$
\begin{aligned}
& \left(\begin{array}{c}
n+j-1 \\
j
\end{array}\right) \int_{t}^{\infty}(-1)^{j+1} \frac{(u-t)^{j-2}}{(j-2) !} \\
& \quad \times f^{(n)}(u) g^{(j)}(u) d u \\
& =(-1)^{j}\left(\begin{array}{c}
n+j-1 \\
j
\end{array}\right) \\
& \quad \times \int_{t}^{\infty} \frac{(s-t)^{j-1}}{(j-1) !} \\
& \quad \times\left(f^{(n+1)} g^{(j)}+f^{(n)} g^{(j+1)}\right)(s) d s
\end{aligned}
$$

for $2 \leq j \leq n$ and $t \geq 0$. Using these equalities and the combinatorial identity

$$
\left(\begin{array}{c}
n+j \\
j
\end{array}\right)+\left(\begin{array}{c}
n+j \\
j+1
\end{array}\right)=\left(\begin{array}{c}
n+1+j \\
j+1
\end{array}\right)
$$


we obtain that

$$
\begin{aligned}
(n+1) & \left(f^{(n)} g^{(1)}\right)(t)+\sum_{j=2}^{n-1}\left(\begin{array}{c}
n+j-1 \\
j
\end{array}\right) \\
& \times \int_{t}^{\infty}(-1)^{j+1} \frac{(u-t)^{j-2}}{(j-2) !} f^{(n)}(u) g^{(j)}(u) d u \\
= & \sum_{j=1}^{n-1}\left(\begin{array}{c}
n+j \\
j
\end{array}\right)(-1)^{j} \\
& \times \int_{t}^{\infty} \frac{(s-t)^{j-1}}{(j-1) !} f^{(n+1)}(s) g^{(j)}(s) d s \\
& +\left(\begin{array}{c}
2 n-1 \\
n-1
\end{array}\right)(-1)^{n+1} \\
& \times \int_{t}^{\infty} \frac{(s-t)^{n-2}}{(n-2) !} f^{(n)}(s) g^{(n)}(s) d s .
\end{aligned}
$$

Similarly, we get

$$
\begin{aligned}
(n+1) & \left(g^{(n)} f^{(1)}\right)(t)+\sum_{j=2}^{n-1}\left(\begin{array}{c}
n+j-1 \\
j
\end{array}\right) \\
& \times \int_{t}^{\infty}(-1)^{j+1} \frac{(u-t)^{j-2}}{(j-2) !} g^{(n)}(u) f^{(j)}(u) d u \\
= & \sum_{j=1}^{n-1}\left(\begin{array}{c}
n+1+j-1 \\
j
\end{array}\right)(-1)^{j} \\
& \times \int_{t}^{\infty} \frac{(s-t)^{j-1}}{(j-1) !} g^{(n+1)}(s) f^{(j)}(s) d s \\
& +\left(\begin{array}{c}
2 n-1 \\
n-1
\end{array}\right)(-1)^{n+1} \\
& \times \int_{t}^{\infty} \frac{(s-t)^{n-2}}{(n-2) !} f^{(n)}(s) g^{(n)}(s) d s .
\end{aligned}
$$

Taking into account that

$$
\begin{aligned}
& 2\left(\begin{array}{c}
2 n-1 \\
n-1
\end{array}\right)(-1)^{n+1} \\
& \quad \times \int_{t}^{\infty} \frac{(s-t)^{n-2}}{(n-2) !} f^{(n)}(s) g^{(n)}(s) d s \\
& =\left(\begin{array}{c}
2 n \\
n
\end{array}\right)(-1)^{n} \\
& \quad \times \int_{t}^{\infty} \frac{(s-t)^{n-1}}{(n-1) !} \\
& \quad \times\left(f^{(n+1)} g^{(n)}+g^{(n+1)} f^{(n)}\right)(s) d s
\end{aligned}
$$

we conclude the proof.

Remark 4. Note that the Leibniz formula holds for functions which belong to larger sets than $\mathcal{S}$.
Given $n \in \mathbb{N}$, we consider the norm $\|\cdot\|_{(n)}$ defined by

$$
\begin{array}{r}
\|f\|_{(n)}:=\frac{1}{(n-1) !} \int_{0}^{\infty} t^{n-1}\left|f^{(n)}(t)\right| d t, \\
f \in \mathcal{S},
\end{array}
$$

and the Banach space $A C^{(n)}$ obtained as the completion of $\mathcal{S}$ in the norm $\|\cdot\|_{(n)}$. A function $f \in A C^{(n)}$ if and only if there exists $f$ has all derivatives up to order $n-1$ satisfying $\lim _{x \rightarrow \infty} x^{k} f^{(k)}(x)=0$, for $0 \leq k \leq n-1$, and the $(n-1)$ th derivative is absolutely continuous with $\int_{0}^{\infty} t^{n-1}\left|f^{(n)}(t)\right| d t<$ $\infty$; see [4, Corollary 3.2]. In particular, $\left(R_{t}^{n}\right)_{t \geq 0} \subset A C^{(n)}$ and $\left\|R_{t}^{n}\right\|_{(n)}=t^{n} / n$ !, for $t \geq 0$; see more details in [4, page 319]. Consider functions $\left(e_{z}\right)_{z \in \mathbb{C}^{+}} \subset A C^{(n)}$ and $\left\|e_{z}\right\|_{(n)}=(|z| / \mathfrak{R} z)^{n}$, where $e_{z}(t):=e^{-z t}$ for $z \in \mathbb{C}^{+}$and $t \geq 0$.

In fact, the space $A C^{(n)}$ is a Banach algebra under pointwise multiplication for $n \in \mathbb{N}$ (see [4, Proposition 3.4]). Applying Proposition 3, a second proof may be given.

\section{Functional Calculus for Operator-Valued Riesz Means}

We introduce the operator-valued Riesz means in an axiomatic way and use them to define a functional calculus which domain is the Banach algebra $A C^{(n)}$. Then, we may associate a closed operator $(A, D(A))$ to operator-valued Riesz means.

Definition 5. For $n \in \mathbb{N}$, one calls operator-valued Riesz mean of degree $n$ any strongly continuous family $\left(G_{t}^{n}\right)_{t \geq 0} \subset \mathscr{B}(X)$ such that it satisfies the following conditions:

(i) $\sup _{t \geq 0}\left(n ! / t^{n}\right)\left\|G_{t}^{n}\right\|<\infty$;

(ii) $\lim _{t \rightarrow \infty}\left(n ! / t^{n}\right) G_{t}^{n}(x)=x$ for $x \in X$;

(iii) the equality

$$
\begin{aligned}
G_{t}^{n} G_{s}^{n}(x)= & \sum_{j=1}^{n}(t-s)^{n-j} \frac{(n+j) !}{n !^{2}}\left(\begin{array}{c}
n \\
j
\end{array}\right) \\
& \times \int_{0}^{s} \frac{(s-u)^{j-1}}{(j-1) !} G_{u}^{n}(x) d u \\
& +\frac{(t-s)^{n}}{n !} G_{s}^{n}(x)
\end{aligned}
$$

holds for $t \geq s \geq 0$ and $x \in X$.

For $n=1$, we get the Fejer family considered in [9, Section 4]. If $\left(G_{t}^{n}\right)_{t>0} \subset \mathscr{B}(X)$ is an operator-valued Riesz mean of degree $n$, then $\left(G_{t}^{m}\right)_{t \geq 0} \subset \mathscr{B}(X)$ is also an operator-valued Riesz mean of degree $m$, where

$$
\begin{aligned}
G_{t}^{m}(x)= & \frac{1}{(m-n-1) !} \\
& \times \int_{0}^{t}(t-s)^{m-n-1} G_{s}^{n}(x) d s, \quad x \in X,
\end{aligned}
$$


for $m>n$. Note that the Riesz function $\left(R_{t}^{n}\right)_{t \geq 0}$ is an operator-valued Riesz mean of degree $n$ in the space $A C^{(n)}$ (Proposition 1) and $\left(f_{n, s}\right)_{s \geq 0}$ is an operator-valued Riesz mean of degree $n$ in the space $L^{1}(\mathbb{R})$ (Remark 2).

Theorem 6. Let $n \in \mathbb{N}$, and let $X$ be a Banach space.

(i) Given an operator-valued Riesz mean of degree n, $\left(G_{t}^{n}\right)_{t \geq 0} \subset \mathscr{B}(X)$, the map $\Phi: A C^{(n+1)} \rightarrow \mathscr{B}(X)$ defined by

$$
\begin{array}{r}
\Phi(f) x:=(-1)^{n+1} \int_{0}^{\infty} f^{(n+1)}(t) G_{t}^{n}(x) d t, \\
x \in X, \quad f \in A C^{(n+1)},
\end{array}
$$

is a bounded functional calculus such that $\Phi\left(R_{t}^{n+1}\right) x=$ $\int_{0}^{t} G_{s}^{n}(x) d s$ and $\lim _{\lambda \rightarrow 0^{+}} \Phi\left(e_{\lambda}\right) x=x$ for $x \in X$.

(ii) Conversely, given a bounded functional calculus $\Phi$ : $A C^{(n)} \rightarrow \mathscr{B}(X)$ such that $\lim _{\lambda \rightarrow 0^{+}} \Phi\left(e_{\lambda}\right) x=x$ for any $x \in X$, there exists an operator-valued Riesz mean of degree $n\left(G_{t}^{n}\right)_{t>0} \subset \mathscr{B}(X)$ such that $\Phi$ is given by formula (24).

Proof. (i) It is clear that expression (24) defines a bounded homomorphism and $\|\Phi\| \leq \sup _{t \geq 0}\left(n ! / t^{n}\right)\left\|G_{t}^{n}\right\|$. Now, we check that $\Phi(f g)=\Phi(f) \Phi(g)$ for $f, g \in A C^{(n+1)}$. Due to condition (i) of Definition 5, we apply the Fubini theorem to obtain that

$$
\begin{aligned}
\Phi(f) \Phi(g) x= & \int_{0}^{\infty} \int_{0}^{t} f^{(n+1)}(t) g^{(n+1)}(s) G_{t}^{n} G_{s}^{n}(x) d s d t \\
& +\int_{0}^{\infty} \int_{0}^{s} f^{(n+1)}(t) g^{(n+1)}(s) G_{t}^{n} G_{s}^{n}(x) d t d s
\end{aligned}
$$

for $x \in X$. Now, we apply condition (iii) of Definition 5 and the equality

$$
\begin{aligned}
& \int_{0}^{\infty} f^{(n+1)}(t) \int_{0}^{t}(t-s)^{n-j} g^{(n+1)}(s) \\
& \quad \times \int_{0}^{s} \frac{(s-u)^{j-1}}{(j-1) !} G_{u}^{n}(x) d u d s d t \\
& =(n-j) !(-1)^{n+1+j} \\
& \quad \times \int_{0}^{\infty} G_{u}^{n}(x) \\
& \quad \times \int_{u}^{\infty} \frac{(s-u)^{j-1}}{(j-1) !} f^{(j)}(s) g^{(n+1)}(s) d s d u
\end{aligned}
$$

for $1 \leq j \leq n$ to get

$$
\begin{aligned}
& \int_{0}^{\infty} \int_{0}^{t} f^{(n+1)}(t) g^{(n+1)}(s) G_{t}^{n} G_{s}^{n}(x) d s d t \\
& =\sum_{j=1}^{n}\left(\begin{array}{c}
n+j \\
j
\end{array}\right)(-1)^{n+1+j} \\
& \quad \times \int_{0}^{\infty} G_{u}^{n}(x) \int_{u}^{\infty} \frac{(s-u)^{j-1}}{(j-1) !} f^{(j)}(s) g^{(n+1)}(s) d s d u \\
& \quad+(-1)^{n+1} \int_{0}^{\infty} f(s) g^{(n+1)}(s) G_{s}^{n}(x) d s .
\end{aligned}
$$

It follows that

$$
\begin{aligned}
\Phi(f) & \Phi(g) x \\
= & (-1)^{n+1} \\
& \times \int_{0}^{\infty}\left(f(s) g^{(n+1)}(s)+g(s) f^{(n+1)}(s)\right) G_{s}^{n}(x) d s \\
& +\sum_{j=1}^{n}\left(\begin{array}{c}
n+j \\
j
\end{array}\right)(-1)^{n+1+j} \\
& \times \int_{0}^{\infty} G_{u}^{n}(x) \int_{u}^{\infty} \frac{(s-u)^{j-1}}{(j-1) !} f^{(j)}(s) g^{(n+1)}(s) d s d u \\
& +\sum_{j=1}^{n}\left(\begin{array}{c}
n+j \\
j
\end{array}\right)(-1)^{n+1+j} \\
& \times \int_{0}^{\infty} G_{u}^{n}(x) \int_{u}^{\infty} \frac{(s-u)^{j-1}}{(j-1) !} g^{(j)}(s) f^{(n+1)}(s) d s d u \\
= & (-1)^{n+1} \int_{0}^{\infty}(f g)^{(n+1)}(s) G_{s}^{n}(x) d s \\
= & \Phi(f g) x,
\end{aligned}
$$

where we have applied formula (12) in the last equality.

We get directly that $\Phi\left(R_{t}^{n+1}\right) x=\int_{0}^{t} G_{s}^{n}(x) d s$, for $x \in$ $X$. Now, take $\varepsilon>0$; then, there exists $t_{0}>0$ such that $\left\|\left(n ! / t^{n}\right) G_{t}^{n}(x)-x\right\| \leq \varepsilon$ for $t>t_{0}$. Note that

$$
\begin{aligned}
& \left\|\Phi\left(e_{\lambda}\right) x-x\right\| \\
& \leq \frac{\lambda^{n+1}}{n !} \int_{0}^{t_{0}} t^{n} e^{-\lambda t}\left\|\frac{n !}{t^{n}} G_{t}^{n} x-x\right\| d t \\
& \quad+\varepsilon \frac{\lambda^{n+1}}{n !} \int_{t_{0}}^{\infty} t^{n} e^{-\lambda t} d t \\
& \leq\left(\sup _{t>0} \frac{n !}{t^{n}}\left\|G_{t}^{n}\right\|+1\right) \frac{\|x\|}{n !} \int_{0}^{t_{0} \lambda} u^{n+1} e^{-u} d u+\varepsilon,
\end{aligned}
$$

and we conclude that $\lim _{\lambda \rightarrow 0^{+}} \Phi\left(e_{\lambda}\right) x=x$ for $x \in X$. 
Now, we check part (ii). We define $G_{t}^{n}:=\Phi\left(R_{t}^{n}\right)$. It is clear that $\left(G_{t}^{n}\right)_{t>0}$ is a strongly continuous family of operators and $G_{0}^{n}=0$. Note that

$$
\frac{n !}{t^{n}}\left\|G_{t}^{n}\right\| \leq \frac{n !}{t^{n}}\|\Phi\|\left\|R_{t}^{n}\right\|_{(n)}=\|\Phi\|, \quad t>0
$$

and the first condition of Definition 5 holds. To check the third condition, note that

$$
\begin{aligned}
G_{t}^{n} G_{s}^{n}(x)= & \sum_{j=1}^{n}(t-s)^{n-j} \frac{(n+j) !}{n !^{2}}\left(\begin{array}{l}
n \\
j
\end{array}\right) \\
& \times \int_{0}^{s} \frac{(s-u)^{j-1}}{(j-1) !} G_{u}^{n}(x) d u \\
& +\frac{(t-s)^{n}}{n !} G_{s}^{n}(x),
\end{aligned}
$$

(for $t \geq s \geq 0$ and $x \in X$ ) where we have applied Proposition 1. The proof of part (ii) of Definition 5 is similar to the proof of part (i) above.

Recall that a holomorphic $C_{0}$-semigroup $(T(z))_{z \in \mathbb{C}^{+}} \subset$ $\mathscr{B}(X)$ is a family of operators such that the map $\mathbb{C}^{+} \ni z \mapsto$ $T(z) x \in X$ is analytic, $\lim _{z>0, z \rightarrow 0} T(z) x=x$, and $T\left(z+z^{\prime}\right) x=$ $T(z) T\left(z^{\prime}\right) x$ for $x \in X$ and $z, z^{\prime} \in \mathbb{C}^{+}$.

Now, we consider $(T(z))_{z \in \mathbb{C}^{+}} \subset \mathscr{B}(X)$ a holomorphic $C_{0^{-}}$ semigroup such that

$$
\|T(z)\| \leq C\left(\frac{|z|}{\mathfrak{R} z}\right)^{\alpha}, \quad z \in \mathbb{C}^{+},
$$

for some $\alpha \geq 0$. There exists a big amount of these semigroups; see, for example, [10], [4, Section 7], [3, Lemma 2.3], [11], and the references therein. Then, the family of operators $\left(G_{t}^{n}\right)_{t \geq 0} \subset \mathscr{B}(X)$ defined by

$$
G_{t}^{n}(x):=\frac{1}{2 \pi i} \int_{\mathfrak{R} z=1} \frac{e^{t z}}{z^{n+1}} T(z) x d z, \quad x \in X
$$

is an operator-valued Riesz mean of degree $n$ for $n>\alpha$. Conversely, given an operator-valued Riesz mean of degree $n,\left(G_{t}^{n}\right)_{t \geq 0} \subset \mathscr{B}(X)$, then the family of operators $(T(z))_{z \in \mathbb{C}^{+}} \subset$ $\mathscr{B}(X)$ defined by

$$
T(z) x:=z^{n+1} \int_{0}^{\infty} e^{-z t} G_{t}^{n}(x) d t, \quad x \in X, z \in \mathbb{C}^{+},
$$

is a holomorphic $C_{0}$-semigroup such that

$$
\|T(z)\| \leq \sup _{t>0} \frac{n !}{t^{n}}\left\|G_{t}^{n}\right\|\left(\frac{|z|}{\mathfrak{R} z}\right)^{n+1}, \quad z \in \mathbb{C}^{+} .
$$

In this case, $\sigma(A) \subset[0, \infty)$ and

$$
(\lambda+A)^{-1} x=(n+1) ! \int_{0}^{\infty} \frac{G_{t}^{n}(x)}{(\lambda+t)^{n+2}} d t, \quad \lambda>0, x \in X,
$$

where the operator $(-A, D(A))$ is the infinitesimal generator of a holomorphic $C_{0}$-semigroup $(T(z))_{z \in \mathbb{C}^{+}} \subset \mathscr{B}(X)$. These results are essentially given in [12, Theorem 3.1] for $n=1,2$ and extended [4, Theorem 4.1] under more general conditions than those considered here.

Recall the family of functions $\left(f_{n, s}\right)_{n \in \mathbb{N}, s \geq 0}$ considered in Section 2. The following result appears in [9, Corollary 4.5] for $n=1$. The proof for $n>1$ may be done directly using formula (10) and is also a consequence of the case $n=1$. Similar result for uniformly bounded cosine functions holds.

Corollary 7. Let $(T(t))_{t \in \mathbb{R}}$ be a uniformly bounded $C_{0}$-group generated by $i A$ on a Banach space $X$. Then, the family of operators $\left(G_{s}^{n}\right)_{s>0}$ defined by

$$
G_{s}^{n}(x):=\int_{-\infty}^{\infty} f_{n, s}(t) T(t) x d s, \quad x \in X,
$$

is an operator-valued Riesz mean of degree $n$ associated to $\left(A^{2}\right)^{1 / 2}$.

\section{Conflict of Interests}

The author declares that there is no conflict of interests regarding the publication of this paper.

\section{Acknowledgments}

The author thanks warmly the careful reading, interesting comments, and valuable suggestions made by an anonymous referee which have led to the improvement of the final version of this paper. The author also thanks Eva Fašangová, Ralph Chill, María Martínez, and José E. Galé for several comments, remarks, and helpful discussions. This research was done in the Laboratoire de Mathématiques at Université Paul Verlaine Metz (France) during a visit of the author. The author has been partially supported by Project MTM2010-16679, DGIFEDER, of the MCYTS, Project E-64, D.G. Aragón, and JIUZ-2012-CIE-12, Universidad de Zaragoza, Spain.

\section{References}

[1] N. Dunford and J. T. Schwartz, Linear Operators, Part III, WileyInterscience, New York, NY, USA, 1988.

[2] R. deLaubenfels, "Unbounded well-bounded operators, strongly continuous semigroups and the Laplace transform," Studia Mathematica, vol. 103, no. 2, pp. 143-159, 1992.

[3] J. E. Galé, P. J. Miana, and D. Müller, "Extensions of wellboundedness and $C^{m}$-scalarity," Integral Equations and Operator Theory, vol. 57, no. 3, pp. 327-337, 2007.

[4] J. E. Galé and T. Pytlik, "Functional calculus for infinitesimal generators of holomorphic semigroups," Journal of Functional Analysis, vol. 150, no. 2, pp. 307-355, 1997.

[5] J. Peetre, New Thoughts on Besov Spaces, Duke University Mathematics Series I, Duke University, Durham, NC, USA, 1976.

[6] W. Trebels, "Some Fourier multiplier criteria and the spherical Bochner-Riesz kernel," Revue Roumaine de Mathématiques Pures et Appliquées, vol. 20, no. 10, pp. 1173-1185, 1975.

[7] P. L. Butzer, R. J. Nessel, and W. Trebels, "Multipliers with respect to spectral measures in Banach spaces and approximation. I. Radial multipliers in connection with Riesz-bounded 
spectral measures," Journal of Approximation Theory, vol. 8, pp. 335-356, 1973.

[8] A. Zygmund, Trigonometric Series, Cambridge University Press, Cambridge, UK, 1959.

[9] E. Fašangová and P. J. Miana, "Hilbert, Dirichlet and Fejér families of operators arising from $C_{0}$-groups, cosine functions and holomorphic semigroups," Semigroup Forum, vol. 80, no. 1, pp. 33-60, 2010.

[10] V. Cachia, "Convergence at the origin of integrated semigroups," Studia Mathematica, vol. 187, no. 3, pp. 199-218, 2008.

[11] M. Hieber, "Integrated semigroups and differential operators on $L^{p}$ spaces," Mathematische Annalen, vol. 291, no. 1, pp. 1-16, 1991.

[12] R. deLaubenfels, "Functional calculus for generators of uniformly bounded holomorphic semigroups," Semigroup Forum, vol. 38, no. 1, pp. 91-103, 1989. 


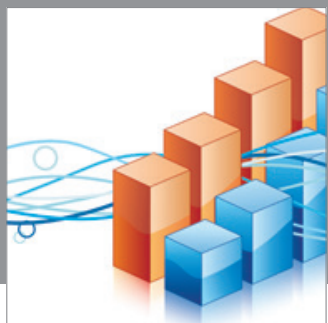

Advances in

Operations Research

mansans

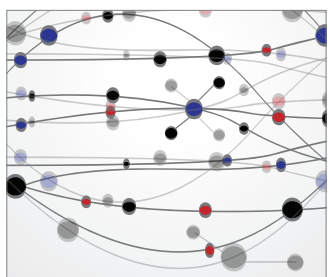

The Scientific World Journal
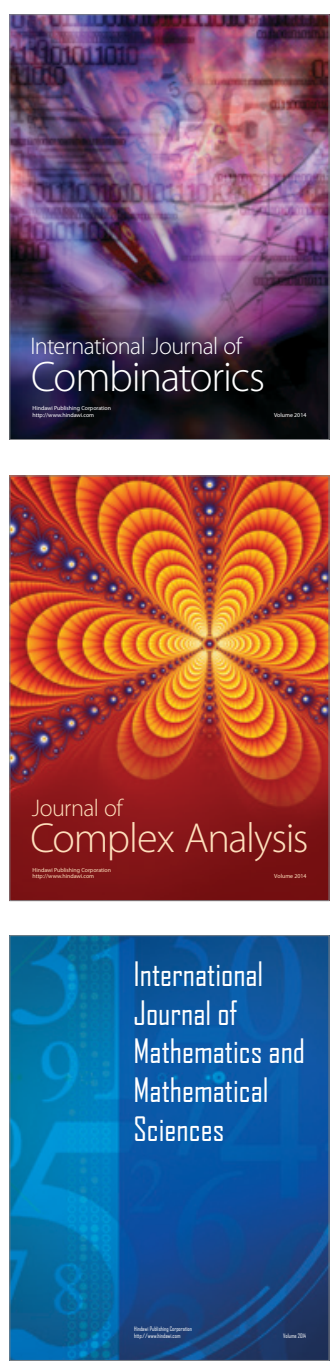
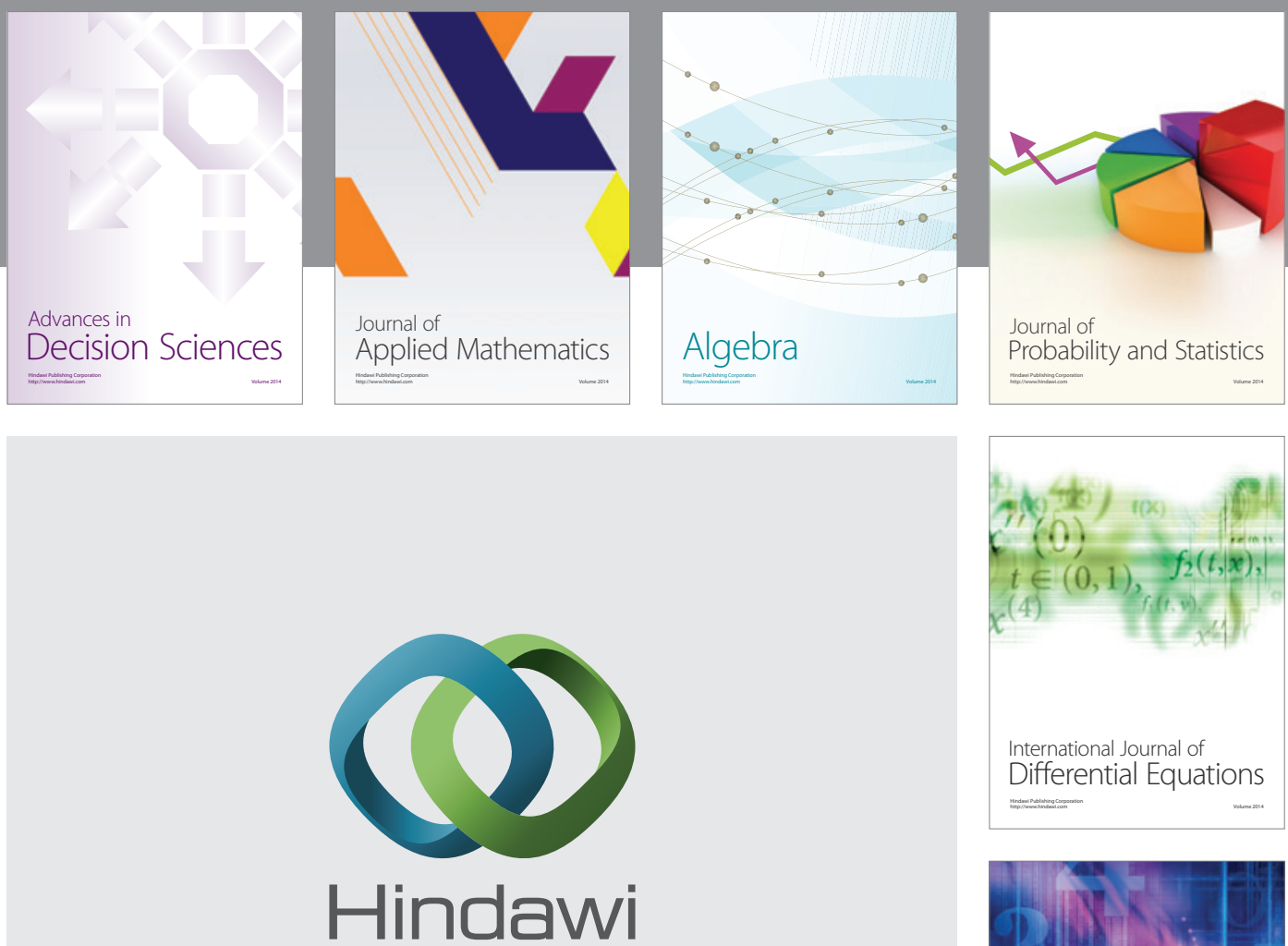

Submit your manuscripts at http://www.hindawi.com
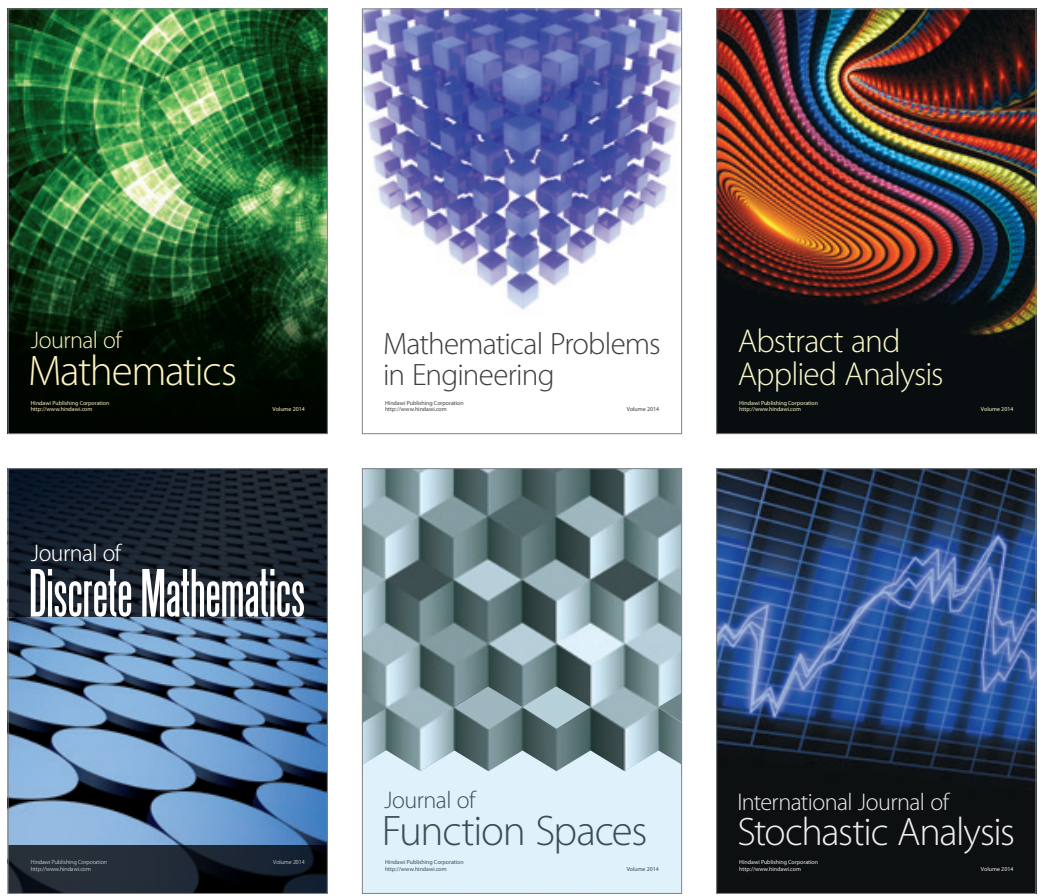

Journal of

Function Spaces

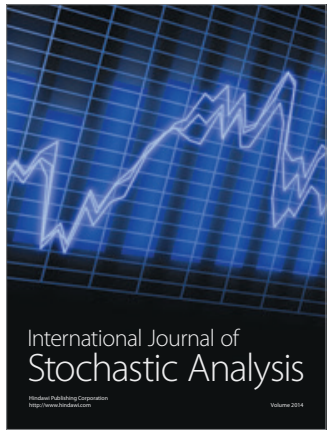

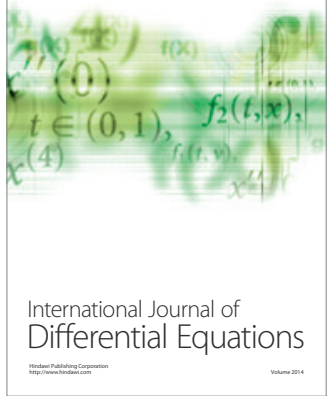
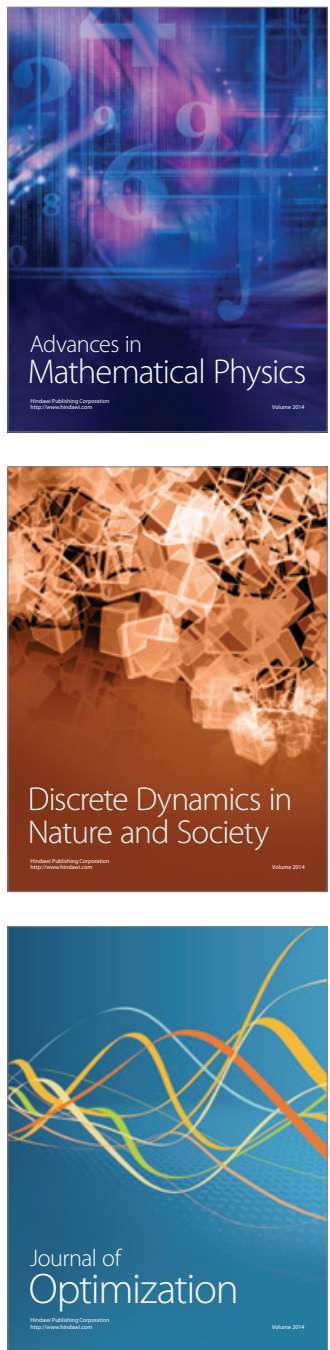